\title{
CREATINA PARA ATLETAS DE ESPORTES COLETIVOS
}

\author{
${ }^{1}$ João Paulo da Costa Oliveira; ${ }^{2}$ Fátima Lúcia Rodrigues Guimarães
}

\begin{abstract}
RESUMO
Introdução: A creatina é um composto sintetizado naturalmente pelo corpo humano. Sua síntese se inicia nos rins e termina no fígado. A creatina é encontrada em maior parte no músculo esquelético, na forma de creatina fosfato, que atua como uma via energética nas atividades intensas de curta duração. Seu consumo não é considerado doping pelo Comitê Olímpico Internacional. Objetivo: Este estudo tem o intuito de investigar benefícios do consumo de creatina nos atletas de modalidades esportivas coletivas com suas diferentes especialidades e características de posição dentro do esporte. Método: foi realizada uma busca nas bases de dados nacionais e internacionais: (SciElo, Google Acadêmico e PubMed), a fim de reunir artigos que através de testes identificassem efeitos da suplementação de creatina em atletas de esportes coletivos. Resultados: A suplementação com creatina apresentou melhora nos testes de força, tiros de velocidade, potência e em saltos verticais. Alguns autores encontraram em seus ensaios um aumento de peso nos indivíduos, o que ocasionou numa diminuição de tempo nos testes de tiros de velocidade. Estes resultados foram encontrados usando a suplementação de creatina apenas, ou em associação com outras substâncias como carboidratos ou cafeína. A creatina também demonstrou ter uma ação antioxidante parcial nos atletas submetidos a testes de força. Discussão: Os resultados encontrados nos testes avaliando o desempenho de força e tiros de velocidade são justificados pelo fato de a creatina, na forma fosforilada, ser uma substância que atua em atividades de alta intensidade e curta duração. Mesmo com ganho de peso, o tempo na execução dos testes de velocidade diminuiu sem afetar o desempenho dos atletas. Conclusão: $O$ efeito ergogênico da creatina nos exercícios de força, mostraram-se eficazes no desempenho esportivo de atletas de esportes coletivos. Essa ação pode ser mais evidente dependendo da função tática que o atleta exerce.
\end{abstract}

Palavras Chaves: Creatina, Suplementação, Auxílio Ergogênico, Desempenho, Esportes Coletivos.

\section{CREATINE FOR COLLECTIVE SPORTS ATHLETES}

\section{ABSTRACT}

Introduction: Creatine is a compound naturally synthesized by the human body. This process of synthesis begins in the kidneys and ends in the liver. The greatest amount of creatine is found in skeletal muscle tissue, as the creatine phosphate form, which acts as an energetic route in intense and short-term exercises. Its consumption is not considered doping by the International Olympic Committee. Objective: This study aimed to investigate the benefits of creatine consumption by athletes of collective sportive modalities. Method: A survey was conducted to search databases in national and international websites (SciElo, Google Scholar and PubMed), to get some articles to identify effects in creatine supplementation in athletes of collective sports. Results: Creatine, in exercise trials, showed some improvement in tests of strength, sprint, power and in vertical jumps. In other results that were found in some exercise trials, creatine caused increase on the weight of the subjects, which resulted in decrease of the time on sprint tests. These results were found using creatine supplementation alone, or in combination with other substances such as carbohydrates or caffeine. Creatine has been shown to have partial antioxidant action in athletes undergoing strength tests. Discussion: The results that were found in the tests that when was evaluated performance in the strength and sprint tests, are justified by the fact that creatine, in phosphorylated form, is a substance that acts in high intensity and short duration and/or interval activities. Weight gain decreased the time in running the sprint tests, but did not affect the performance of the athletes. Conclusion: The ergogenic effect of creatine on strength, intense and short duration activities is shown to be effective on the sportive performance of athletes of collective sports. This action may be more evident depending on the tactical function that the athletes exercise.

Keywords: Creatine, Supplementation, Ergogenic Aid, Performance, Collective Sports.

${ }^{1}$ Centro Universitário São Miguel - UNISÃOMIGUEL ${ }^{2}$ Universidade de Pernambuco - UPE

E-mail: oliveira.joaopc@gmail.com 


\section{INTRODUCÃO}

A creatina foi descoberta em 1832, pelo cientista Michel Eugene, o qual observou o acúmulo desta em músculos esqueléticos de lobos selvagens que eram mais ativos fisicamente quando comparados com outros da mesma espécie (REZENDE, 2003). A creatina, ácido a-metil guanidino acético, é uma amina sintetizada naturalmente pelo corpo humano a partir dos aminoácidos glicina, arginina e metionina (PERALTA e AMANCIO, 2002). A síntese desta amina começa no rim com a glicina e o grupo guanidino da arginina. Essa reação é catalisada pela enzima amidinotransferase, produzindo o guanidinoacetato, que é transportado pela corrente sanguínea até o fígado, onde reage com o grupo metil da metionina por ação da enzima metiltransferase (HALLIWELL, 2006), (NELSON E COX, 2014) produzindo a creatina (GUALANO, 2008).

Após essas reações, a creatina é direcionada ao músculo esquelético, onde ficam armazenados $95 \%$ do seu total no corpo humano. Coração, cérebro, retina e testículos também armazenam, porém, numa proporção menor (ZANELLI, 2015).

A creatina é mais encontrada na sua forma fosforilada, creatina fosfato (CF), que é a molécula de creatina unida a um grupo fosfato. A CF participa de um processo fisiológico de transferência de energia, denominado sistema imediato de ressíntese de adenosina trifosfato (ATP), ou simplesmente sistema ATP-CP, que funciona nos segundos iniciais da contração muscular. Neste sistema, a quebra da CF libera a energia necessária para fosforilar à adenosina difosfato (ADP), gerando uma nova molécula de ATP que serve de suporte energético para as células do corpo humano (AOKI, 2004).

A Creatina também pode ser obtida de forma exógena por meio do consumo de peixes e carnes bovinas, ou na forma de suplemento alimentar (PERALTA e AMANCIO, 2002). Nesta última, a creatina costuma estar monohidratada ou micronizada, sendo formas mais simples de obter auxílio ergogênico nas práticas esportivas (PANTA e FILHO, 2015). A forma micronizada possui partículas menores, dissolve-se melhor em líquidos, que favorece uma maior absorção intestinal. Já a creatina monohidratada é um pó branco solúvel em água, sendo a mais comum, mais barata e mais estudada em artigos, e composta por $88 \%$ de creatina e $12 \%$ de água, porém, de menor dissolução.
Existem diversos mecanismos que auxiliam na melhoria do desempenho no esporte, e os recursos ergogênicos são apontados como ferramentas importantes para auxiliar na concretização deste objetivo (CONFORTIN, SÁ e WILDNER, 2016). Como o uso da creatina não é considerado dopping pelo Comitê Olímpico Internacional (COI), esta substância se popularizou ainda mais entre atletas profissionais e amadores, que tinham o objetivo de melhorar os resultados nas competições (PERALTA e AMANCIO, 2002).

Nos jogos Olímpicos de Barcelona (1992) foi observado que os corredores de alta velocidade obtiveram um melhor desempenho nas atividades de força e potência, relacionada ao uso da suplementação de creatina (FRANCO e MARIANO, 2009). Além disso, a procura pela creatina como suplemento também acontece com o objetivo de melhora no desempenho em treinos de atividades intervaladas e de alta intensidade, por considerar que a creatina seja um produto ergogênico para atividades com essas características (KNAPIK et al., 2016).

Neste contexto, o presente trabalho buscou em bancos de dados periódicos que avaliassem, principalmente, os efeitos da ingestão de creatina, podendo estar associada a outras substâncias que ajudariam num potencial de absorção ou ação da creatina, no desempenho de atletas, para identificar efeitos desta suplementação nos testes físicos que avaliassem os componentes de força, velocidade, resistência entre outros.

\section{MÉTODO}

A presente pesquisa trata-se de uma revisão bibliográfica a fim de identificar benefícios da suplementação de creatina em atletas. Para isso foi realizada uma busca nas seguintes bases de dados nacionais e internacionais: SciELO (www.scielo.br), PubMed (www.ncbi.nlm.nih.gov/pubmed/) e na plataforma de busca Google Acadêmico (https://scholar.google.com.br). Os termos usados foram: creatina, suplementação creatina, creatina esportes, creatina suplementação atletas, ergogênicos esportes. Termos em inglês correspondentes aos citados anteriormente também foram usados nas pesquisas. A busca e seleção dos artigos contemplados ocorreram entre outubro de 2017 e novembro de 2019.

Foram inseridos na presente pesquisa ensaios clínicos usando humanos que utilizaram a creatina como principal meio de intervenção, usando os protocolos de saturação (20-25g por dia durante 5-7 dias, ou $0,3 \mathrm{~g} / \mathrm{kg}$ durante 5-7 dias), que é uma fase de 
doses mais altas, e/ou manutenção ( $3-5 \mathrm{~g}$ por dia, ou $0,03 \mathrm{~g} / \mathrm{kg}$ ), uma fase de dose mais baixas. Apesar destes meios de intervenção ter sido mais encontrados, outros protocolos de suplementação não foram descartados. Também foram inseridos artigos de revisão bibliográfica. Foram excluídos estudos de conclusão de curso: monografias, dissertações e/ou teses. Ensaios com indivíduos não treinados também não foram incluídos.

\section{RESULTADOS E DISCUSSÃO}

Tabela 1. Efeito ergogênico da creatina em atletas de esportes coletivos.

\begin{tabular}{|c|c|c|c|c|c|c|}
\hline Autor & $\begin{array}{c}\text { Dose de } \\
\text { carregamento }\end{array}$ & $\begin{array}{c}\text { Dose de } \\
\text { carregamento }\end{array}$ & $\begin{array}{c}\text { Testes } \\
\text { aplicados }\end{array}$ & Resultados & Esporte & $\begin{array}{c}\text { Substância } \\
\text { adicional }\end{array}$ \\
\hline $\begin{array}{l}\text { LARSON- } \\
\text { MEYER et al., } \\
\text { (2000). }\end{array}$ & $\begin{array}{l}7,5 \mathrm{~g} \text { de } \mathrm{Cr} 2 \text { vezes } \\
\text { ao dia, durante } 7 \\
\text { dias. }\end{array}$ & $\begin{array}{l}5 \mathrm{~g} \text { de } \mathrm{Cr} \text {, dose } \\
\text { única diária por } \\
12 \text { semanas. }\end{array}$ & $\begin{array}{l}\text { Teste de força, } \\
\text { agachamento } \\
\text { livre e supino } \\
\text { reto. }\end{array}$ & $\begin{array}{lr}\text { Aumento } & \text { da } \\
\text { força, } & \text { massa } \\
\text { magra e gordura } \\
\text { livre. }\end{array}$ & Futebol & ---- \\
\hline $\begin{array}{l}\text { PRADO et al., } \\
(2007) \text {. }\end{array}$ & $\begin{array}{l}25 \mathrm{~g} \text { de } \mathrm{Cr}, 5 \text { vezes } \\
\text { ao dia durante } 5 \\
\text { dias. }\end{array}$ & $\begin{array}{l}5 \mathrm{~g} \text { de } \mathrm{Cr} \text { por } \\
\text { dia, durante } 5 \\
\text { dias. }\end{array}$ & $\begin{array}{l}\text { Testes de } \\
\text { velocidade. }\end{array}$ & $\begin{array}{lr}\text { Aumento } & \text { no } \\
\text { desempenho } & \text { nos } \\
\text { testes } & \text { de } \\
\text { velocidade. } & \end{array}$ & $\begin{array}{l}\text { Basquete } \\
\text { bol }\end{array}$ & ---- \\
\hline $\begin{array}{l}\text { CHILIBECK, } \\
\text { MAGNUS e } \\
\text { ANDERSON } \\
\text { (2007). }\end{array}$ & ---- & $\begin{array}{lr}0,1 \mathrm{~g} / \mathrm{kg} & \mathrm{de}+ \\
0,7 \mathrm{~g} / \mathrm{kg} & \text { de } \\
\text { glicose } & \text { ou } \\
0,8 \mathrm{~g} / \mathrm{kg} & \text { de } \\
\text { glicose. } & \end{array}$ & $\begin{array}{l}\text { Aeróbicos; } \\
\text { anaeróbicos ( } \\
\text { tiros de } \\
\text { velocidade); } \\
\text { resistência } \\
\text { muscular. }\end{array}$ & $\begin{array}{l}\text { O grupo creatina } \\
\text { teve maior no de } \\
\text { repetições no } \\
\text { supino, leg press, } \\
\text { e foi melhor nos } \\
\text { testes aeróbicos. }\end{array}$ & Rúgbi & Glicose \\
\hline $\begin{array}{l}\text { LAMNTAGNE } \\
\text { L-LACASSE; } \\
\text { NANDON e } \\
\text { GOULET } \\
(2011) .\end{array}$ & $\begin{array}{l}\text { Dia } 1 \text { até dia } 4, \\
80 \mathrm{~g} \text { de glicose, } \\
40 \mathrm{~g} \text { de sacarose e } \\
20 \mathrm{de} \mathrm{Cr} \text {, dia } 5 \text { e } 6 \\
40 \mathrm{~g} \text { de glicose, } \\
20 \mathrm{~g} \text { de sacarose e } \\
10 \mathrm{~g} \text { de Cr. }\end{array}$ & $\begin{array}{l}22 \text { dias de } 20 \mathrm{~g} \\
\text { de glicose, } 10 \mathrm{~g} \\
\text { de sacarose e } \\
5 \mathrm{~g} \text { de Cr. }\end{array}$ & $\begin{array}{l}\text { Salto vertical e } \\
\text { salto com } \\
\text { deslocamento. }\end{array}$ & $\begin{array}{lr}\text { O grupo } & \mathrm{Cr} \\
\text { melhorou } & \text { a } \\
\text { capacidade } & \text { nos } \\
\text { saltos, } & \\
\text { comparado } & \text { ao } \\
\text { placebo. } & \end{array}$ & Voleibol & $\begin{array}{l}\text { Glicose } \\
\text { sacarose. }\end{array}$ \\
\hline $\begin{array}{l}\text { DEMINICE et } \\
\text { al., (2013). }\end{array}$ & $\begin{array}{l}0,3 \mathrm{G} / \mathrm{Kg} \text { de } \mathrm{Cr} \\
\text { durante } 7 \text { dias. }\end{array}$ & ----- & $\begin{array}{l}\text { Testes de } \\
\text { velocidade. }\end{array}$ & $\begin{array}{lr}\text { Melhorou } & 0 \\
\text { desempenho nos } \\
\text { testes } & \text { de } \\
\text { velocidade. } & \end{array}$ & Futebol & ----- \\
\hline $\begin{array}{l}\text { CLAUDINO et } \\
\text { al., (2014). }\end{array}$ & $\begin{array}{l}20 \mathrm{~g} \text { de } \mathrm{Cr} 4 \text { vezes } \\
\text { ao dia durante } 7 \\
\text { dias. }\end{array}$ & $\begin{array}{lr}5 \mathrm{~g} \text { de } \mathrm{Cr} \text { por dia } \\
\text { durante } & 6 \\
\text { semanas. } & \end{array}$ & $\begin{array}{l}\text { Saltos } \\
\text { verticais. }\end{array}$ & $\begin{array}{l}\text { Maior } \\
\text { desempenho no } \\
\text { grupo que } \\
\text { consumiu } \\
\text { creatina. }\end{array}$ & Futebol & ---- \\
\hline $\begin{array}{l}\text { CONFORTIN, } \\
\text { SÁ } \\
\text { WILDNER } \\
(2016) .\end{array}$ & $\begin{array}{l}0.03 \mathrm{G} / \mathrm{kg} \text { de } \mathrm{Cr} \text { de } \\
\text { dextrose } 4 \text { vezes } \\
\text { ao dia durante } 5 \\
\text { dias. }\end{array}$ & ---- & $\begin{array}{l}\text { Testes de } \\
\text { velocidade. }\end{array}$ & $\begin{array}{l}\text { Piora } \\
\text { resultado } \\
\text { testes } \\
\text { velocidade. }\end{array}$ & Futebol & Dextrose \\
\hline $\begin{array}{l}\text { YANEZ-SILVA } \\
\text { et al., (2017). }\end{array}$ & ----- & $\begin{array}{l}0.03 \mathrm{G} / \mathrm{kg} \text { de } \mathrm{Cr} \\
\text { durante } 14 \text { dias. }\end{array}$ & $\begin{array}{l}\text { Teste } \\
\text { anaeróbio, } \\
\text { Wingate. }\end{array}$ & $\begin{array}{l}\text { Melhorou o pico } \\
\text { de potência e } \\
\text { potência máxima. }\end{array}$ & Futebol & ---- \\
\hline
\end{tabular}


Larson-Meyer et al., (2000) investigaram os efeitos da suplementação de creatina ou placebo na força muscular e na composição corporal em atletas de futebol do sexo feminino, num ensaio clínico de 13 semanas. Neste estudo, foram utilizados dois protocolos de intervenção com creatina, $7,5 \mathrm{~g}$ de creatina ou placebo duas vezes ao dia por uma semana, e $5 \mathrm{~g}$ de creatina ou placebo por dia até o fim do protocolo. Ao final do protocolo, foi observado que as atletas que usaram creatina demonstraram grande melhoria nos movimentos de supino e agachamento livre, em comparação ao grupo que usou placebo. A massa magra, medidas por absorciometria com raios- $x$, composta por ossos e músculos, e a gordura livre aumentaram com o treinamento, que foi o fator principal, e não devido à suplementação de creatina como esperado.

Em Prado et al., (2007), jogadores de basquetebol que receberam $25 \mathrm{~g}$ por dia, de placebo (maltodextrina) ou creatina, durante cinco dias, e posteriormente $5 \mathrm{~g}$ por dia nos cinco dias subsequentes, aumentaram o peso corporal, no entanto, melhoraram a capacidade de tiros de velocidade repetidos. Foram aplicados testes de 6 tiros de velocidade consecutivos de 30 metros, com 2 minutos de pausa entre uma execução e outra. Os atletas que usaram creatina tiveram um melhor desempenho nos tiros de velocidade quando comparados com o grupo placebo.

Nos resultados encontrados, com relação ao tempo de execução dos tiros de velocidade (30 metros), verificou-se alteração em relação ao período pré e póssuplementação de creatina. $O$ quarto, quinto e o sexto tiro de velocidade, dos componentes do grupo creatina, tiveram seu tempo de execução, em segundos, reduzido em $8,5 \%$, $10 \%$ e $9 \%$ (Pré 4 $7,51 \pm 0,37 ; 5 \div 7,76 \pm 0,39 ; 6^{\circ}$ $7,69 \pm 0,41$ e Pós $4^{\circ} 6,87 \pm 0,26 ; 5^{\circ}$ 6,96 $\pm 0,36 ; 6^{\circ}$ $6,97 \pm 0,28)$, respectivamente, comparado com o primeiro tiro sem suplementação. Os componentes do grupo creatina comparados ao grupo placebo tiveram os tempos do terceiro, quarto quinto e sexto tiros de velocidade reduzidos em $11 \%, 12 \%, 11 \%$ e $12 \%$ (Pla 3 ㅇ $7,70 \pm 0,40 ; \quad 4$ ㅇ $7,73 \pm 0,46 ; \quad 5$ ㅇ $7,90 \pm 0,52 ; 607,86 \pm 0,44$ e $\mathrm{Cr} 3^{\circ}$ 7,47 $\pm 0,47 ; 4^{\circ}$ $\left.7,51 \pm 0,37 ; \quad 5^{\circ} \quad 7,76 \pm 0,39 ; \quad 6^{\circ} \quad 7,69 \pm 0,41\right)$, respectivamente, comparado com o primeiro tiro sem suplementação.

Chilibeck, Magnus e Anderson (2007) fez uma intervenção de 8 semanas com 19 jogadores de rúgbi, num teste randomizado (duplo-cego), dividindo os atletas em dois grupos creatina $(\mathrm{Cr})$, com 10 atletas $(6$ atacantes e 4 defensores), e placebo (Pla) com 9 atletas (5 atacantes e 4 defensores). Para o grup $\mathrm{Cr}$, ele forneceu $0,1 \mathrm{~g} / \mathrm{kg} / \mathrm{dia}$ de creatina monohidratada com $0,7 \mathrm{~g} / \mathrm{kg} / \mathrm{dia}$ de glicose. Já para o grupo Pla, foi fornecida a dose de $0,8 \mathrm{~g} / \mathrm{kg} / \mathrm{dia}$ de glicose. A partir disso, os atletas foram submetidos a testes aeróbicos, tiros de velocidade anaeróbicos e treinos de resistência muscular, duas vezes por semana, com sessões de até 2 horas, e mais 80 minutos de jogo por semana.

Para avaliar a resistência muscular, foi realizado o máximo de repetições no supino reto e no leg press com $75 \%$ de 1 RM. Já para avaliar a resistência aeróbica, foi aplicado o teste leger shuttle-run. Ao final da intervenção, - grupo $\mathrm{Cr}$ teve um maior número de repetições $(5,18 \pm 1,4$ repetições, $21,1 \% \pm$ $4,9 \%)$ comparado com o grupo Pla $(0,9 \pm 2$ repetições, $8,8 \% \pm 10 \%$ ), sendo $p<0,05$. Já 0 aumento no teste aeróbico foi de $8,8 \%$ no $\mathrm{Cr}$ e $9,7 \%$ no Pla.

Lamontagne- Lacasse (2011) avaliou 8 jogadores de voleibol no salto vertical e no salto com deslocamento. Os atletas foram divididos em 2 grupos com 4 participantes cada, sendo um $\mathrm{Cr}$ e outro Pla. A intervenção durou 28 dias, sendo que nos 4 dias iniciais, os atletas tomaram 4 porções de creatina ou placebo com 3 horas de intervalo entre as doses. No dia 5 e 6 , os atletas tomaram 2 porções por dia, com 3 horas de intervalo, e nos 22 dias restantes da intervenção, tomaram 1 dose após a sessão de treino. Cada dose continha $20 \mathrm{~g}$ de dextrose, $10 \mathrm{~g}$ de sacarose, $300 \mathrm{ml}$ de água, e para o grupo $\mathrm{Cr}, 5 \mathrm{~g}$ de creatina monohidradata. Foi realizado um teste inicial, onde o indivíduo tinha 5 tentativas com 1 minuto de intervalo, para fazer o salto com deslocamento e o vertical o mais alto possível. O teste para avaliar a capacidade do salto dos atletas, consistia em 10 séries de 10 saltos, com 3 segundos de intervalos entre os saltos e 2 minutos entre as séries. Ambos os grupos aumentaram a altura repetida do salto vertical $(6,6 \% \pm 2 \%$ no $\mathrm{Cr}$, e $4,7 \% \pm 2,3 \%$ no Pla). Nas séries 3-10, o grupo $\mathrm{Cr}$ melhorou a capacidade de salto $(2,8 \% \quad \pm 1,5 \%, \quad p=0,12)$ significativamente em relação ao grupo Pla $(1,9 \% \pm 1,8 \%, 0=0,33)$. A capacidade repetida de salto vertical, aumentou em média $3,8 \mathrm{~cm} \pm$ $1,3 \mathrm{~cm}$ no grupo $\mathrm{Cr}$ e $2,5 \mathrm{~cm} \pm 0,9 \mathrm{~cm}$ no grupo $\mathrm{Pla}$, nas 10 séries.

A investigação de Deminice et al., (2013), também no desporto do futebol, testaram-se seis tiros de velocidade de corrida, com tiros de 35 metros e intervalo de 10 segundos de descanso entre tiros, em grupos de atletas que foram suplementados 
com $0,3 \mathrm{~g} / \mathrm{kg}$ de creatina ou placebo em barras, por sete dias, num ensaio duplo-sego randomizado. A utilização da creatina foi capaz de aumentar a concentração plasmática de creatina (201\%) em comparação com o grupo placebo.

Os valores em Watts de potência média (PLA 461,4 \pm 48.1 e CR: 531,2 \pm 94,2, $p<0,05)$, potência máxima (PLA 631,3 $\pm 46,8$ e CR 749,8 $\left.\pm 112,3^{*} p<0,05\right)$ e potência mínima (PLA 314,1 $\pm 66,7^{*}$ e CR 396,1 \pm 67,5* $\mathrm{p}<0,05)$, medidos pelo tempo de cada tiro dos 35 metros, foram maiores no grupo suplementado por creatina do que no grupo de placebo, mostrando que a creatina foi importante na melhora do desempenho nos tiros de velocidade na corrida dos jogadores de futebol que participaram da pesquisa.

As análises de Claudino et al., (2014) foram específicas para a potência dos músculos de membros inferiores no desempenho de saltos verticais. Nos testes foi utilizada uma placa de força strain-gauge em atletas de elite de um time de futebol. Foi observado em atletas que receberam $20 \mathrm{~g} /$ dia de monohidrato de creatina, durante uma semana, dividida em quatro doses diárias iguais, seguido por uma única dose diária de 5 $\mathrm{g}$ para as próximas seis semanas.

Houve uma melhora do desempenho nos testes de saltos verticais quando comparado com os atletas que receberam solução placebo. A comparação dos resultados entre grupos mostrou que ambos os grupos não alcançaram alturas nos saltos verticais significativamente diferentes apesar de valores terem sido maiores no grupo que usou creatina. A variação percentual no grupo placebo foi de - $0,7 \%$, menor que no grupo creatina, onde a variação percentual foi de $2,4 \%$. Porém, os resultados encontrados apontam que não houve diferença significativa $(p=0,23)$.

Já Confortin, Sá e Wildner (2016) investigaram, também em jogadores de futebol, se a suplementação com creatina poderia retardar a fadiga muscular com base na capacidade de realizar tiros de velocidade de alta intensidade nos treinamentos e nos jogos. No entanto, jogadores que foram tratados com placebo por 5 dias (4 sachês de $10 \mathrm{~g}$ de talco farmacêutico por dia), e realizaram 0 teste de tiros de velocidade repetidos (sete tiros de 20 metros separados por 25 segundos de recuperação passiva), obtiveram melhores tempos do que quando foram submetidos ao mesmo protocolo, 8 dias após o término do primeiro, substituindo 0 tratamento placebo por mistura de $5 \mathrm{~g}$ de creatina $+5 \mathrm{~g}$ de dextrose, constatando que a suplementação com creatina aumentou 0 índice de fadiga.

Yáñez-Silva et al., (2017) usaram atletas de futebol realizando testes de 30s no Teste Anaeróbico de Wingate (TAW), investigando o pico de potência, a potência média e índices de fadiga. O protocolo de suplementação usado nesse período foi de $0,03 \mathrm{~g} / \mathrm{kg}$ de creatina ou placebo (maltodextrina) num período de 14 dias. Os resultados encontrados indicaram que uma baixa dose de suplementação oral em curto prazo de monohidrato de creatina $\left(0,03 \mathrm{~g} / \mathrm{kg}^{-1}\right.$ por dia em 14 dias) parece ter beneficiado a produção de energia em jogadores de futebol, e sem nenhum efeito colateral adverso. Pois, os jogadores de futebol suplementados por creatina apresentaram o pico de potência e potência média melhorada nos testes, após a baixa dose de suplementação de creatina num curto período de tempo.

\& Apesar de alguns resultados demonstrarem que alguns atletas ganharam peso durante o período de suplementação, o que seria um fator que impossibilitaria o uso da creatina por atletas, esse aumento de peso não impediu os atletas de manterem o seu rendimento mesmo com o ganho de peso.

$O$ efeito ergogênico da creatina em atividades de alta intensidade e curta duração já foi comprovado em diversos estudos como mostra (TERENZI, 2013). Os protocolos mais usados nos ensaios clínicos são 0 de carregamento, que consiste numa dose mais alta (de $20 \mathrm{~g}$ até $25 \mathrm{~g}$ ou $0,3 \mathrm{~g} / \mathrm{kg}$ ) de creatina, num período curto de 5 até 7 dias, e 0 protocolo de manutenção que é, geralmente, usado em ensaios clínicos de longo prazo. O protocolo de manutenção são doses de $3 \mathrm{~g}$ até $5 \mathrm{~g}$ de creatina ou $0,03 \mathrm{~g} / \mathrm{kg}$ (TERENZI, 2013).

A dose mais baixa de creatina usada nos protocolos de manutenção assemelha-se à quantidade consumida diariamente nos alimentos somada a produção endógena (GUALANO et al., 2007). O efeito ergogênico da creatina nos esportes com as características citadas acima se dá ao fato de a suplementação com creatina possibilitar o aumento do reservatório orgânico deste composto em até 20\% (LOON et al., 2003). Além disso, há estudos que mostram que esse aumento pode ser de até $50 \%$ em indivíduos vegetarianos (PERALTA, AMANCIO, 2002). Com isso vários estudos se destinaram a estudar os efeitos da suplementação no rendimento esportivo.

Os relatos de benefícios e vantagens exibidos nos artigos fazem da creatina um dos 


\section{ESPORTES COLETIVOS E CREATINA}

suplementos alimentares mais procurados no meio esportivo para melhoria do desempenho, nas mais diversas modalidades, tanto por atletas amadores quanto por atletas de alto nível (WADDINGTON et al, 2005; MANSON et al., 2001). Os motivos que levam os atletas a suplementarem com creatina são aumento da força, aumento da massa magra, melhorar a recuperação entre as séries e até melhorar a ressíntese de glicogênio muscular durante a fase de carregamento (RANCHORDAS, DAWSON, RUSSELL, 2017).

Um dos efeitos relatados é o aumento de massa magra e peso corporal. Esse fato foi justificado em muitos estudos apenas pela retenção hídrica decorrentes do uso do suplemento. Estes indicam que a suplementação de creatina aumenta o peso corporal na primeira semana de carregamento de creatina devido à sua característica osmótica. Contudo, estudos questionam se as mudanças nos conteúdos intracelulares de água podem influenciar a tradução de proteínas contráteis e justificar parte do real ganho de massa magra (ZANELLI et al., 2015). Esse fato pode trazer uma resposta negativa ao rendimento do atleta nas suas práticas esportivas, pois existe uma crença de que este aumento prejudicaria o desempenho. Entretanto, ainda não existem evidências suficientes para comprovar este efeito adverso da suplementação de creatina (PRADO et al., 2007). Nos resultados encontrados por Prado (2007) houve um aumento de peso, mas tal aumento não afetou o desempenho dos atletas.

O uso da creatina também vem sendo associado a outras substâncias como, por exemplo, a cafeína. Doses baixas de cafeína demonstram efeito ergogênico, e esses níveis podem ser obtidos facilmente com alimentos presentes da dieta cotidiana (TARNOPOLSKI, 2010). A creatina e a cafeína não exibem interações farmacocinéticas, interações em que um dos fármacos modifica a cinética de outro administrado concomitantemente, quando ingeridas em conjunto, e são pensadas para melhorar 0 desempenho através de mecanismos independentes, o que originou interesse no potencial de suplementação combinada (TREXLER et al., 2017).

Trexler et al., (2017) num ensaio clínico com 54 indivíduos treinados, aplicou o protocolo de $20 \mathrm{~g}$ de creatina por dia durante cinco dias associado a 300mg em média de cafeína $(3-5 \mathrm{mg} / \mathrm{kg})$, e submeteu esses indivíduos a treinos de uma repetição máxima (1RM) no supino e leg press, e testes de tiros de velocidade. No fim do estudo observaramse efeitos principais significativos nos exercícios de 1RM. Os testes de tiros de velocidade, que ocorreram 10 minutos após os testes de força, não mostraram resultados significantes, pois o pico máximo de esforço e o trabalho total tiveram uma insignificante variação. Os resultados nos treinos de força foram semelhantes aos atingidos por LarsonMeyer et al. (2000), que não utilizou substância associada com a creatina, o que tornaria desnecessário o uso da cafeína.

O exercício físico é uma condição que exerce influência no balanço entre ações prooxidantes e mecanismos de defesa antioxidante. A síntese de oxidantes é mais acentuada no exercício físico intenso, pois a demanda energética é aumentada. Um desbalanço deste sistema redox em excesso pode provocar estresse oxidativo, que resulta da produção exacerbada de agentes oxidantes capazes de causar danos celulares e várias doenças (COQUEIRO et al, 2017).

Com a intensificação da utilização de CF e de ATP pela fibra muscular durante esse tipo de exercício, ocorre ativação simultânea do ciclo de degradação de purinas (ciclo de Lowenstein), devido ao aumento na concentração intramuscular de adenosina monofosfato (AMP). As principais consequências da ativação dessa via são a produção paralela de amônia, hipoxantina, xantina, urato e de espécies reativas de oxigênio (HALLIWELL, 2006).

Estudos realizados com suplementação de creatina previamente ao exercício intenso resultaram em queda, tanto na produção de amônia como na de hipoxantina (SOUZA JUNIOR e PEREIRA, 2008). Esse possível efeito, seria favorável para o controle da fadiga muscular e fornecer um melhor rendimento para os consumidores da creatina, mas isso não conseguiu ser constatado no estudo de Confortin, Sá e Wildner (2016), onde os atletas tiveram um aumento no índice de fadiga e tiveram um rendimento pior após uso da creatina. Percário et al., (2012) num estudo mais detalhado, recrutou 26 atletas de handebol de alto nível e dividiram em grupos onde um dos grupos usaria $20 \mathrm{~g}$ de creatina (GC) por dia durante cinco dias e $5 \mathrm{~g}$ por mais 27 dias, outro grupo usaria placebo (GP) e outro não participaria do tratamento (GST).

Durante o protocolo de suplementação os integrantes dos grupos foram submetidos a testes de 1RM nos exercícios de supino, crucifixo no banco inclinado, puxada alta, remada baixa, elevação lateral, rosca direta, 
agachamento e cadeira extensora. Nos resultados não houve mudanças significativas no peso, massa magra e massa gorda em nenhum dos grupos após os protocolos de suplementação. $\mathrm{Na}$ análise dos marcadores de estresse oxidativo houve um aumento significativo de $61 \%$ sobre o valor médio póstreinamento de ácido úrico para o GC, quando comparado ao GP e GST. Não houve diferença nas substâncias reativas ao ácido tiobarbitúrico (TBARS). Não houve diferença das amostras dos grupos nos exames de CPK, creatinina e ureia após o exercício.

Os valores de status totais antioxidantes diminuíram consideravelmente no GC em comparação com o GP e GST. Além disso, o GC demonstrou uma significativa redução de $46 \%$ nos valores de status totais antioxidantes ao comparar 0 tempo pré e pós-suplementação. Com esse resultado, Percário et al. (2012) trouxe um efeito pouco relatado ao uso de creatina, mas que pode trazer benefícios aos atletas.

O uso da suplementação de creatina também foi associado à dextrose, pois a melhor prática de suplementação de creatina é quando a ingestão desta é associada com a

\section{CONCLUSÃO}

O consumo de creatina como suplemento alimentar, substância ergogênica não considerada como doping pelo Comitê Olímpico Internacional, tem se mostrou eficaz na melhoria do desempenho esportivo, porém em condições específicas do exercício físico, como por exemplo, atividades curtas, intensas e com períodos de recuperação. Este efeito é justificado pelo fato da creatina atuar como uma reserva de ATP na musculatura esquelética, que poderia potencializar a regeneração de ATP. Estes efeitos podem trazer benefícios na melhora do desempenho dos atletas em atividades que exigem força, de carboidrato, pois assim pode-se aumentar o acúmulo de creatina em até $60 \%$ quando comparada ao uso de creatina apenas (BACURAU, 2007). Os resultados encontrados por Confortin, Sá e Wildner (2016), não apresentaram melhora no desempenho durante os testes de tiros de velocidade nos atletas.

A creatina foi associada aos casos de disfunção renal em atletas (KUEHL, GOLDBERG, ELLIOT, 1998). Essa ideia foi sustentada por muito tempo com a hipótese de que aumento da ingestão de creatina aumentaria os níveis de creatinina que ocasionaria numa disfunção renal. Gualano et al., (2008) numa revisão bibliográfica apresentou que a suplementação em doses altas ou baixas de creatina aumentaram os níveis de creatinina, mas não foram suficientes para causar uma disfunção renal. Ele ainda concluiu que apesar dos casos indicando que a creatina possa prejudicar disfunção renal, não foram encontradas evidências sustentáveis de que a suplementação de creatina possa apresentar riscos à saúde do consumidor.

potência e velocidade nas modalidades esportivas em equipe, de acordo com a função tática e do posicionamento em campo do atleta.

Sobre os efeitos da creatina associada a outras substâncias, que intensificam a sua absorção ou que atuam ajudando o seu potencial de ação, como um carboidrato ou cafeína, por exemplo, são necessários mais estudos que possam comprovar essa efetividade potencial dessas substâncias agregadas à creatina, assim como seu, até então, possível potencial antioxidante.

\section{REFERÊNCIAS}

AOKI, M. S. Suplementação de Creatina e Treinamento de Força: Efeito do Tempo de Recuperação Entre as Séries. Revista Brasileira de Ciência e Movimento. Brasília v. 12 n. 4 p. 39 44, Dezembro 2004.

BACURAU, R. F. Nutrição e Suplementação Esportiva, 5 edição, São Paulo, editora Phorte, 2007. CHILIBECK, P. D.; MAGNUS, C.; ANDERSON, M. Effect of In-Season Creatine Supplementation on Body Composition and Performance in Rugby Union Football Players. Applied Physiology, Nutrition, and Metabolism, v. 32, n. 6, p. 1052-1057, 2007.

CLAUDINO, J. G. et al. Creatine Monohydrate Supplementation on Lower-Limb Muscle Power in Brazilian Elite Soccer Players. Journal of the International Society of Sports Nutrition, v. 11, n. 32. Junho, 2014. 
CONFORTIN, F. G.; SÁ, C. A.; WILDNER, P. P. Avaliação da Creatina Associada à Dextrose Como Suplemento Nutricional Ergogênico Sobre a Performance de Atletas de Futebol. Revista Brasileira de Nutrição Esportiva, São Paulo, v. 10, n. 56, p. 136-144, Março/Abril, 2016.

COQUEIRO, A. Y. et al; Creatina Como Antioxidante em Estados Metabólicos Envolvendo Estresse Oxidativo. Revista Brasileira de Prescrição e Fisiologia do Exercício, São Paulo, v.11, n. 64, p. 128-137. Janeiro/Fevereiro, 2017.

DEMINICE, R. et al. Effects of Creatine Supplementation on Oxidative Stress and Inflammatory Markers After Repeated-Sprint Exercise in Humans. Nutrition, Burbank, v. 29, n. 9, p, 1127-1132, Setembro. 2013.

FRANCO, G. L.; MARIANO, A. C. Suplementação de Creatina e o Efeito Ergolítico da Cafeína. Revista Brasileira de Nutrição Esportiva, São Paulo, v. 3, n. 13, p. 3, 2009.

GUALANO, B. et al. A Suplementação de Creatina Prejudica a Função Renal? Revista Brasileira de Medicina do Esporte, São Paulo, v. 14, n. 1, p. 68-73, Janeiro/Fevereiro, 2008.

HALLIWELL, B. Oxidative Stress and Neurodegeneration: Where Are We Now? Journal of Neurochemistry, v. 97, n. 6, p. 1634-1658, 2006.

KNAPIK, J. J. et al. Prevalence of Dietary Supplement Use by Athletes: Systematic Review and Meta-Analysis. Sports Medicine, Auckland, v. 46, n. 1, p.103-123, Janeiro, 2016.

KUEHL, K.; GOLDBERG, L.; ELLIOT, D. Renal insufficiency after creatine supplementation in a college football athlete. Medicine and Science in Sports and Exercise, Indianapolis, n. 30, p. 103106, 1998 .

LAMONTAGNE-LACASSE, M.; NADON, R.; GOULET, E. D. B. Effect of Creatine Supplementation on Jumping Performance in Elite Volleyball Players. International Journal of Sports Physiology and Performance, Champaign, v. 6, n.4, p. 525-533, Dezembro, 2011.

LARSON-MEYER, D. et al. The Effect of Creatine Supplementation on Muscle Strength and Body Composition During Off-Season Training in Female Soccer Players. Journal of Strength \& Conditioning Research, Colorado Springs, v.14, n.4, Novembro, 2000.

LOON, L. J. C. V. et al. Effects of Creatine Loading and Prolonged Creatine Supplementation on Body Composition, Fuel Selection, Sprint and Endurance Performance in Humans. Clinical Science, Londres, v.104, n. 2, p. 153-162, Fevereiro, 2003.

MANSON, M. A. et al. Use of Nutritional Supplements by High School Football and Volleyball Players. The lowa Orthopaedic Journal, lowa City, n. 21, p. 43-48, 2001.

NELSON, D. L.; COX, M. M. Princípios de Bioquímica de Lehninger. 6 ed. Porto Alegre: Artmed, 2014.

PANTA, R.; FILHO, J. N. S. Efeitos da Suplementação de Creatina na Força Muscular de Praticantes de Musculação: Uma Revisão Sistemática. Revista Brasileira de Nutrição Esportiva, São Paulo, v. 9, n. 54, p. 518-524, Novembro/Dezembro, 2015.

PERALTA, J; AMANCIO, O. M. S. A creatina como suplemento ergogênico para atletas. Revista de Nutrição. Campinas, v. 15, n. 1, p. 83-93, Janeiro/Abril, 2002.

PERCARIO, S. et al. Effects of Creatine Supplementation on Oxidative Stress Profile of Athletes. Journal of the International Society of Sports Nutrition, v. 9, n. 56, Dezembro, 2012.

PRADO, R. G. et al. Suplementação de Creatina Potencializa o Desempenho de Tiros de velocidade Consecutivos em Jogadores de Basquetebol. Revista Brasileira de Ciência e Movimento, Brasília, v. 15, n. 1, p. 23-28, 2007. 
RANCHORDAS, M. K.; DAWSON, J. T.; RUSSELL, M. Practical Nutritional Recovery Strategies for Elite Soccer Players When Limited Time Separates Repeated Matches. Journal of the International Society of Sports Nutrition, v. 14, n. 35, Setembro, 2017.

REZENDE, A. R. A. Suplementação de Creatina no Treinamento de Musculação e Influência no Aumento da Massa Muscular. Vida \& Saúde, Juiz de Fora, v. 1, n. 2, 2003.

SOUZA JUNIOR, T. P.; PEREIRA, B. Creatina: Auxílio Ergogênico Com Potencial Antioxidante? Revista de Nutrição. Campinas, v. 21, n. 3, p. 349-353, Maio/Junho, 2008.

TARNOPOLSKI, M. A. Caffeine and Creatine Use in Sport. Annals of Nutrition and Metabolism, Ottawa, v. 57, n, 2, p. 1-8, 2010.

TERENZI, G. A Creatina Como Recurso Ergogênico em Exercícios de Alta Intensidade e Curta Duração: Uma Revisão Sistemática. Revista Brasileira de Nutrição Esportiva, São Paulo. v. 7. n. 38. p. 91-98, Março/Abril, 2013.

TREXLER, E. T. et al. Effects of Coffee and Caffeine Anhydrous Intake During Creatine Loading. Journal of Strength and Conditioning Research, v.30, n. 5, p. 1438-1446, Maio, 2017.

WADDINGTON, I. et al. Drug Use in English Professional Football. British Journal of Sports Medicine, Londres, v. 39, n. 4, Abr. 2005.

YÁÑEZ-SILVA, A. et al. Effect of Low Dose, Short-Term Creatine Supplementation on Muscle Power Output in Elite Youth Soccer Players. Journal of the International Society of Sports Nutrition, v. 14, n. 5, Fevereiro, 2017.

ZANELLI, J. C. S. et al. Creatina e Treinamento Resistido: Efeito na Hidratação e Massa Corporal Magra. Revista Brasileira de Medicina do Esporte, São Paulo, v. 21, n. 1, p. 27-31, Janeiro/Fevereiro, 2015. 